\title{
X-Ray Ptychography with a Laboratory Source
}

\author{
Darren J. Batey $\odot,{ }^{1, *}$ Frederic Van Assche, ${ }^{2}$ Sander Vanheule, ${ }^{2}$ Matthieu N. Boone $\odot{ }^{2}$ \\ Andrew J. Parnellø, ${ }^{3}$ Oleksandr O. Mykhaylyk $\odot,{ }^{4}$ Christoph Rau, ${ }^{1}$ and Silvia Cipiccia $\odot^{1,5}$ \\ ${ }^{1}$ Diamond Light Source, Harwell Science and Innovation Campus, Fermi Avenue, Didcot OX11 ODE, United Kingdom \\ ${ }^{2} U G C T-R P$, Department of Physics and Astronomy, Ghent University, Ghent 9000, Belgium \\ ${ }^{3}$ Department of Physics and Astronomy, University of Sheffield, Sheffield S3 7RH, United Kingdom \\ ${ }^{4}$ Soft Matter Analytical Laboratory, Department of Chemistry, University of Sheffield, Sheffield S3 7HF, United Kingdom \\ ${ }^{5}$ Department of Medical Physics and Biomedical Engineering, University College London, London WC1E 6BT, United Kingdom
}

(Received 5 January 2021; revised 19 March 2021; accepted 8 April 2021; published 12 May 2021)

\begin{abstract}
X-ray ptychography has revolutionized nanoscale phase contrast imaging at large-scale synchrotron sources in recent years. We present here the first successful demonstration of the technique in a small-scale laboratory setting. An experiment was conducted with a liquid metal-jet x-ray source and a single photoncounting detector with a high spectral resolution. The experiment used a spot size of $5 \mu \mathrm{m}$ to produce a ptychographic phase image of a Siemens star test pattern with a submicron spatial resolution. The result and methodology presented show how high-resolution phase contrast imaging can now be performed at smallscale laboratory sources worldwide.
\end{abstract}

DOI: 10.1103/PhysRevLett.126.193902

Ptychography is a coherent scanning-diffraction imaging technique that produces quantitative images at resolutions beyond the imaging performance of conventional, lensbased, microscopy systems [1]. Ptychography is now routinely applied at x-ray synchrotron sources across the world, obtaining highly sensitive, quantitative, images at the highest spatial resolutions, down to tens of nanometers [2-7]. Until now, the high level of coherence required for x-ray ptychography has limited the application of the technique to high brilliance sources such as synchrotron and, more recently, FEL facilities [8]. It was recently postulated that the new generation of $\mathrm{x}$-ray laboratory sources may have sufficient brilliance to conduct a ptychographic experiment, given the correct experimental setup [9]. We present here a demonstration of such an experiment and the first proof of concept for far field $\mathrm{x}$-ray ptychography performed using an x-ray laboratory source.

A ptychography scan consists of recording $2 \mathrm{D}$ intensity patterns downstream from a sample that is irradiated by a localized spot of coherent radiation. The 4D ptychographic dataset is built up by scanning the sample relative to the beam to a series of overlapping positions. It is possible to record and subsequently invert the data to retrieve the complex refractive index of the object at wavelength limited resolutions across an

Published by the American Physical Society under the terms of the Creative Commons Attribution 4.0 International license. Further distribution of this work must maintain attribution to the author(s) and the published article's title, journal citation, and DOI. extended field of view [10-14]. The success of the inversion step in extracting the phase relies strongly on the stability of the instrumentation and coherent properties of the beam. The coherence manifests itself in interference fringes that hold the relative phase information. The coherent fraction of a beam is related to the lateral (i.e., spatial) and longitudinal (i.e., temporal) coherence. The former is determined by the photon energy and the effective source size-how well confined the source of radiation is laterally in space. The latter is determined by the source bandwidth-how well confined the source of radiation is in wavelength, or longitudinally in space. The level of coherence of an instrument can be described in terms of brilliance. Brilliance is directly proportional to the spatial and temporal coherence. Typical brilliance of third generation light sources is of the order of $10^{20}$ photons s${ }^{-1} \mathrm{~mm}^{-2} \mathrm{mrad}^{-2} 0.1 \%$ B.W.

In a recent work, we used a detuned synchrotron source and a hyperspectral $\mathrm{x}$-ray detector to demonstrate the feasibility of broadband spectroscopic x-ray ptychography [9]. Because of the specific setup, the brilliance of the synchrotron source was reduced to approximately $3 \times 10^{11}$ photons s $\mathrm{mm}^{-2} \mathrm{mrad}^{-2} 0.1 \%$ B.W.. State of the art high brilliance $\mathrm{x}$-ray laboratory sources based on a liquid metal-jet (LMJ) approach this level of brilliance [15]. X-ray ptychography using such a source is therefore feasible, as presented in the following.

The experiment was designed and conducted to explore the possibility of ptychographic imaging in a laboratory setting. The data were collected at the University of Sheffield Soft Matter AnalyticaL Laboratory (SMALL) [16,17], with the portable ptychography end station from I13-1 of Diamond Light Source and a hyperspectral detector from Ghent 


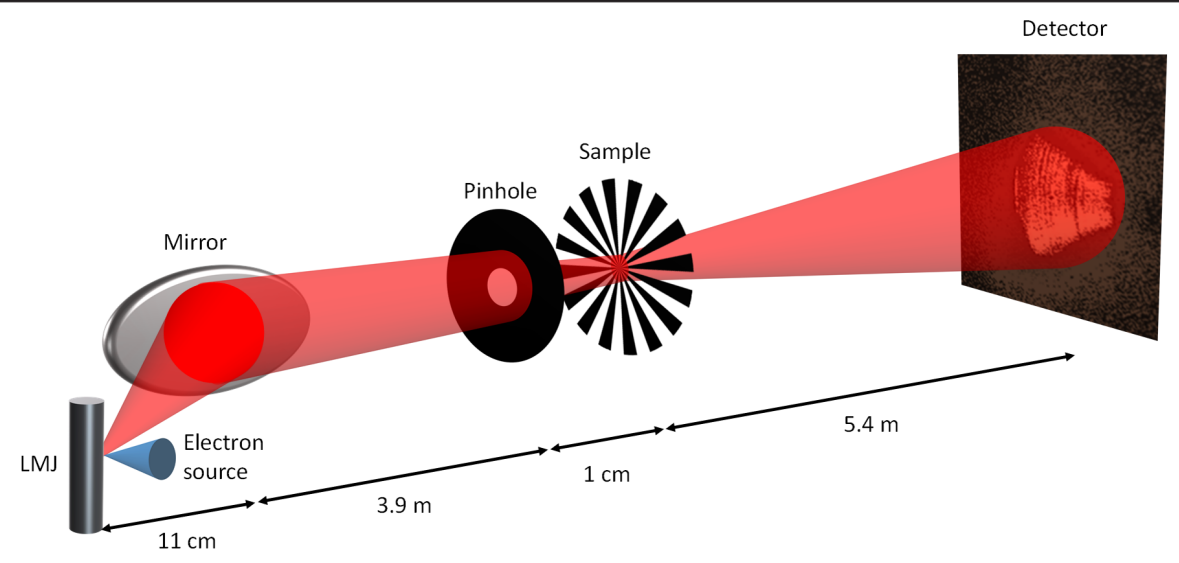

FIG. 1. Experimental configuration. Ptychography setup (not to scale) showing the experimental layout as implemented at SMALL, Sheffield, UK.

University $[18,19]$. The $\mathrm{x}$-ray source is an Excillum liquid gallium metal jet (LMJ), which has a brilliance of approximately $5 \times 10^{11}$ photons $\mathrm{s}^{-1} \mathrm{~mm}^{-2} \mathrm{mrad}^{-2} 0.1 \% \mathrm{~B}$.W. [15], one order of magnitude higher than conventional microfocus sources $[20,21]$. The experimental setup is shown in Fig. 1.

The x-ray beam is generated using a JXS-D2-001 liquid metal-jet laboratory source modified to a higher power performance (Excillum AB, Kista, Sweden) with gallium as anode material. The focal spot size of the source can be varied within a relatively wide range between $5 \mu \mathrm{m}$ and more than $50 \mu \mathrm{m}$, by tuning the projection of the electron beam on the gallium jet stream with a set of electromagnetic lenses. For this experiment, the focal spot size was set to a nominal value of $5 \mu \mathrm{m}$. A three-dimensional single reflection multilayered ellipsoidal mirror (FOX3D 11-600 $\mathrm{Ga}$, Xenocs, Grenoble, France) is used to focus the X-ray beam. The center of the mirror is located $11 \mathrm{~cm}$ downstream of the X-ray source, coinciding with the first mirror focus. The resulting beam is slightly converging, with the second mirror focus located approximately $5.2 \mathrm{~m}$ downstream of the mirror. Because of the chromatic behavior of the mirror reflectivity, the mirror also acts as a spectral bandpass filter, enhancing the relative intensity of the $9.25 \mathrm{keV} \mathrm{K}$-alpha emission line of gallium by drastically reducing the Bremsstrahlung continuum spectrum.

The pnCCD based color X-ray camera (SLcam) [22] was used to measure the diffraction patterns. The detector has a physical pixel pitch of $48 \mu \mathrm{m}$, and an active area of $264 \times 264$ pixels. The system was operated at a readout speed of 400 fps. The in-house developed software SpeXiDAQ [23] was used for camera control and readout as well as raw data processing. The energy resolution of the SLcam is approximately $144 \mathrm{eV} \mathrm{FWHM}$ at the manganese $\mathrm{K}$-alpha peak and the center of mass accuracy is better than $10 \mathrm{eV}$ [24]. The SLcam captures raw frames containing only a few photon events per frame. The raw frames are subsequently preprocessed using a cluster-finding algorithm and subsequent rebinning of the retrieved events into a 3D data cube (two spatial dimensions and one spectral dimension). Because of this processing method, charge sharing effects do not deteriorate the spectral response and subpixel accuracy can be achieved [25]. The spectrum has been generated by integrating the photon counts in each of the $5 \mathrm{eV}$ energy bin datasets. The spectrum recorded is shown in Fig. 2: the escape peak of the K-alpha line in the silicon bulk and the double and triple photon pileup of the $\mathrm{K}$ alpha are visible, beside the main gallium $\mathrm{K}$-alpha and K-beta peaks. The source spectrum has been retrieved by adding the counts of the escape peak, those of the double pileup $(\times 2)$ and of the triple pileup $(\times 3)$ to the K-alpha peak.

The portable ptycho-scope end station developed at the I13-1 branch line of the Diamond Light Source was used for positioning the sample and the pinhole (Fig. 1). The ptycho-scope consists of two 3-axis SLC2430 piezo stages (SmarActs GmbH, Oldenburg, Germany), one for the pinhole and one for the sample. The stages are controlled with PYTHON data collection software connected to an MCS control box over an RS232 protocol.

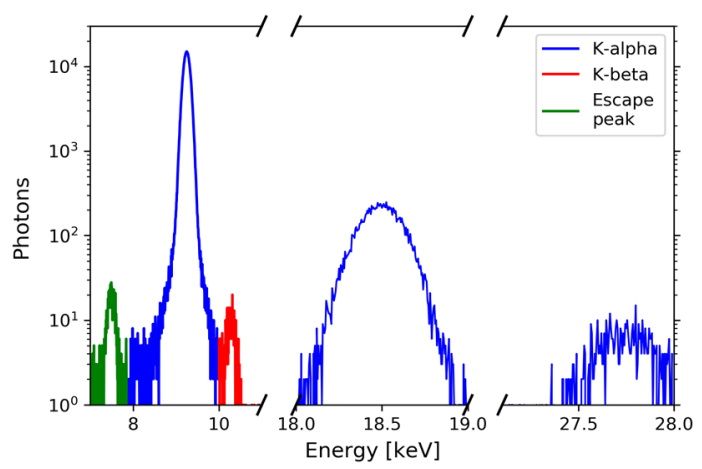

FIG. 2. The X-ray source spectrum as recorded with the SLcam. The gallium K-alpha peak $(9.25 \mathrm{keV})$ along with the double pileup (18.5 keV) and triple pileup peaks $(27.75 \mathrm{keV})$ are shown in blue. The K-alpha escape peak in silicon $(7.51 \mathrm{keV})$ is shown in green and the K-beta $(10.26 \mathrm{keV})$ is shown in red. 
The software scans the position point by point, triggers the detector through a USB-BNC connection, and uses the detector ready status for synchronizing the motion with the detector readout and beam status.

The experiment consisted of a ptychographic scan of a Siemens star test pattern. The Siemens star is a $500 \mathrm{~nm}$ thick gold structure deposited on a silicon nitride membrane with an outer spoke separation of $4 \mu \mathrm{m}$ and an inner spoke separation of $50 \mathrm{~nm}$. An area of $400 \mu \mathrm{m}^{2}$ was scanned during the experiment. The instrument was set up with a $5 \mu \mathrm{m}$ diameter, $50 \mu \mathrm{m}$ thick, tungsten pinhole placed $4 \mathrm{~m}$ from the source. During the experiment, a flux through

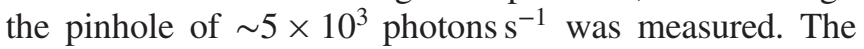
sample was placed $1 \mathrm{~cm}$ downstream of the pinhole and scanned in the plane perpendicular to the optical axis of the beam on a square grid of $20 \times 20$ steps with step size of $1 \mu \mathrm{m}$, following a snakelike trajectory. The detector was placed downstream of the sample at $9.4 \mathrm{~m}$ from the source. Vacuum pipes were placed between the sample and the detector as well as between the mirror and the pinhole to reduce the air absorption and scattering. The detector exposure at each point was $140 \mathrm{~s}$, with a single scan taking 16 hours in total.

Each diffractogram is formed from the raw detector data using SpeXiDAQ [23]. The software processes and splits the data by time and energy into different datasets. The timebased splitting was used for assessing the spatial resolution, the energy-based splitting for investigating the spectral properties. Typical diffractograms are shown in Figs. 4(e) and $4(\mathrm{f})$.

The ptychographic data were reconstructed with 500 iterations of the ePIE operator [12] available in PtyREX [26], where the set of intensity measurements are inverted into an image of the object. The image reconstruction process takes a model of the experiment, including knowledge of the illumination conditions and scanning coordinates along with the recorded intensity measurements, and applies physical constraints in order to solve for the unknown sample. Here, the illumination was initially modeled as a convergent beam of $1 \mathrm{mrad}$ full angle and a defocus of $10 \mathrm{~mm}$. The convergence angle is calculated from the beam on the detector, and the defocus was chosen to produce a $5 \mu \mathrm{m}$ spot creating a balance between the true focal distance of the mirror and the beam width imposed by the pinhole. The scanning coordinates are taken from the requested values of the SmarAct motors. The reconstruction algorithm is capable of dealing with source instability, experimental errors, and signal degradation due to noise and decoherence. The beam intensity was monitored during the acquisition by integrating the flux received on the detector. The intensity variations, shown in Fig. 3(a), are a manifestation of the source instabilities. The source appears to fluctuate across the first 100 positions, with a significant sudden drop in intensity at position 131 of the scan. Scan positions 131 and 132 were removed from the
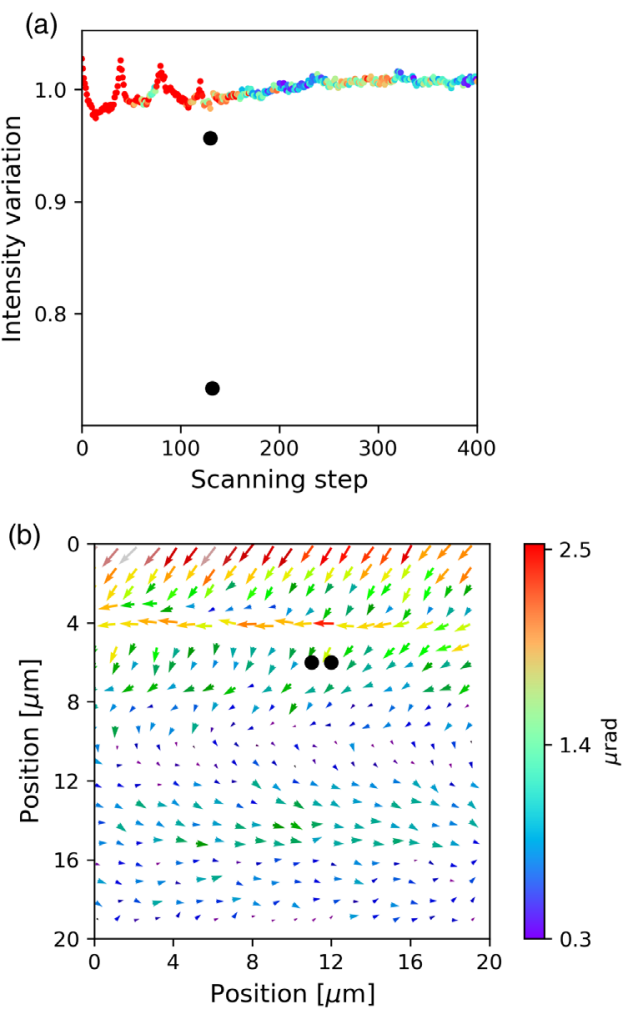

FIG. 3. Source and illumination stability. (a) Fluctuation of total intensity measured by integrating the total flux on the detector during the scan. The value is normalized by the mean value. (b) Beam position and tilt correction as recovered during the PtyREX reconstruction. The origin of the arrow represents the recalculated position, the direction of the arrow represents the direction of the tilt correction, and the length of the arrow is proportional to the modulus of the angular tilt. The color map of both (a) and (b) represents the modulus of the angular tilt at each scan position, highlighting the correlation between intensity fluctuations and angular tilt corrections. The two points removed during the reconstruction are marked as black dots both in (a) and (b).

data prior to the reconstruction (see Fig. 3). The source fluctuations translate into point-to-point instabilities at the sample plane and correspond to either a translation or a tilt, or a combination of the two in the beam profile. PtyREX employs a scan correction built on the annealing method of Maiden et al. [27], but is extended to also accommodate angular variations in the incident beam within the same update step. The position and tilt correction applied during the reconstruction are shown in Fig. 3(b).

The impact of the source properties, detector readout, and beam-sample positions on the reconstruction quality, was investigated. In order to understand the effects of each element and to extract the maximum image quality, a multidimensional parameter sweep was performed on the HPC cluster of Diamond Light Source. The parameters included were the number of source states [28,29], number of scan correction trials [27], detector threshold levels, and 
(a)

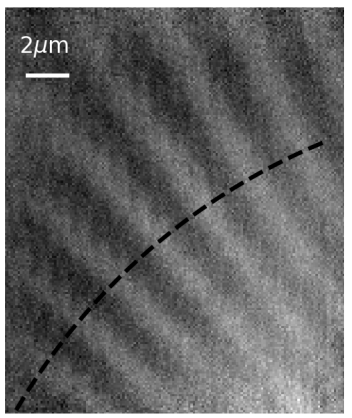

(c)

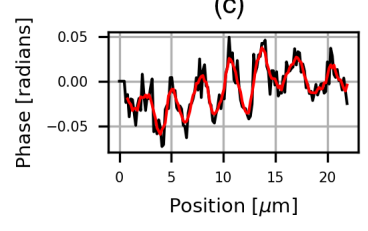

(e)

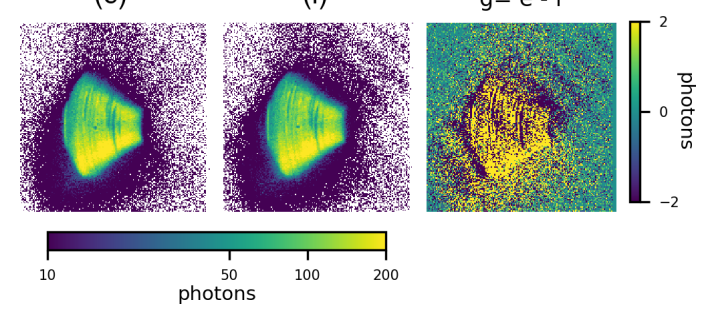

FIG. 4. The ptychography reconstruction. (a) Phase image of the Siemens star test target with a reconstructed pixel size of $116 \mathrm{~nm}$. (b) Modulus of the beam profile at the sample plane. (c) Line plot from the dashed black line in (a). The raw data are represented by the solid black line and the 10 pixel moving average is represented by the solid red line. (d) Fourier ring correlation of the two split exposure reconstructions showing $1.08 \mu \mathrm{m}^{-1}$ spatial frequency (corresponding to $930 \mathrm{~nm}$ ). Two diffractograms recorded in two different scan positions are shown in (e) and (f). (g), the difference (e)-(f), shows clearly the interference fringes.

the bandwidth of the diffraction data. Each parameter permutation was executed on the split and complete exposure data, allowing for a quantitative comparison of the resolution.

The energy bandwidth was investigated and matched to the resolution achievable from the experimental conditions (flux and geometry). The data shown in Fig. 4 were produced using a single output bin ranging from $8.75 \mathrm{keV}$ to $9.75 \mathrm{keV}$.

To quantify the attained resolution, the acquired dataset was divided (in time) into two half datasets to perform a Fourier Ring Correlation (FRC) analysis. The correlation between the two half datasets was compared to a half-bit information threshold, using an implementation based on van Heel et al. [30]. The splitting was done by alternately assigning a camera time-frame series to the odd or even dataset. Since the frame interval is very short $(2.5 \mathrm{~ms})$ compared to the expected timescale of source fluctuations, these half datasets can be considered to be statistically independent measurements of the same source-objectcamera system, including its fluctuations. The correlation in Fourier space was determined over 65 rings.

The detector also made it possible to postprocess the data for different bandwidths. Two datasets, of bandwidth $200 \mathrm{eV}$ (matching the detector energy resolution at the gallium $\mathrm{K}$-alpha energy) and $1 \mathrm{keV}$ around the $\mathrm{K}$-alpha line, were generated. The natural spectral width of the K-alpha line is a few electronvolts, and the distance between K-alpha(1) and $\mathrm{K}$-alpha(2) is $17 \mathrm{eV}$, hence the recorded bandwidth is determined by the detector energy resolution. Increasing the bandwidth of the data analyzed from $200 \mathrm{eV}$ to $1 \mathrm{keV}$ increases the contribution of the Bremsstrahlung background. The theoretical resolutions achievable for $200 \mathrm{eV}$ and $1 \mathrm{keV}$ bandwidths are $100 \mathrm{~nm}$ and $540 \mathrm{~nm}$, respectively [31]. Conversely, the reconstructions of the experimental data showed a worse resolution for the narrower bandwidth (1200 $\mathrm{nm}$ for $200 \mathrm{eV}$ and $930 \mathrm{~nm}$ for $1 \mathrm{keV}$ ), suggesting that the experiment is photon limited.

The best reconstruction, Fig. 4(a), was obtained with $1 \mathrm{keV}$ bandwidth. Both reconstructions included the correction for the source position and direction. These corrections were essential to compensate for longterm instabilities of the source during the acquisition. Figure 4(b) confirms a beam profile of $5 \mu \mathrm{m}$ in extent. A line profile across the reconstructed phase image of the object shows that the spokes are well resolved [Fig. 4(c)]. The obtained FRC curve is shown in Fig. 4(d), as well as the half-bit threshold curve used to determine the attained resolution. The crossover point of the two curves lies at $1.08 \mu \mathrm{m}^{-1}$, corresponding to a resolution of $930 \mathrm{~nm}$, a factor of more than 5 beyond the spot size at the sample.

Performing lab-based x-ray ptychography has required advances in lab sources [20] and detector technologies [19]. The high brilliance of the LMJ has provided the coherent flux required for the ptychography technique. The hyperspectral detector has been required to characterize spectrally the source and assess the temporal coherence.

Our analysis of the results suggests that the experiment was limited by the photon statistics and point-to-point stability of the source. The effect of the latter was mitigated via the reconstruction algorithm that modeled the source shift and direction. The use of different or additional optical components for focussing the $\mathrm{x}$-ray beam to the sample could help to better harness the coherent flux, increasing the photon statistics and reducing the sensitivity to long term source instabilities.

We have demonstrated that it is possible to perform x-ray ptychography with a LMJ source and have shown how to perform ptychography in a laboratory setting, releasing to the laboratory environment a technique otherwise confined to synchrotron facilities.

The resolution achieved in this first experiment is comparable to other lab-based phase contrast techniques, 
such as in-line phase contrast and edge illumination (e.g., Refs. [32-35]). The experimental breakthrough achieved with a LMJ is a first step toward expanding $\mathrm{x}$-ray ptychography to other bright compact light sources: from inverse Compton scattering [36], to laser-plasma based [37] and compact storage rings [38]. The higher brilliance promised by the new sources [39] along with novel coherence enhancement strategies [40], are likely to allow ptychography to compete and outperform the highest resolution laboratory techniques such as X-ray ultra microscopy[41,42] and those based on Zernike phase contrast [43].

O. O. M. thanks EPSRC for the capital equipment grant (EP/M028437/1) to purchase the laboratory-based beam line used for the data collection. The Research Foundation Flanders (FWO; Grant No. G0A0417N) Ghent University Special Research Fund (BOF-UGent; Grant No. BOF17GOA-015) are acknowledged for the financial support to this work. The authors acknowledge Dr. Christian David for the design and production of the Siemens star test pattern.

*Corresponding author darren.batey@diamond.ac.uk

[1] H. M. L. Faulkner and J. M. Rodenburg, Phys. Rev. Lett. 93, 023903 (2004).

[2] J. M. Rodenburg, A. C. Hurst, A. G. Cullis, B. R. Dobson, F. Pfeiffer, O. Bunk, C. David, K. Jefimovs, and I. Johnson, Phys. Rev. Lett. 98, 034801 (2007).

[3] M. Dierolf, A. Menzel, P. Thibault, P. Schneider, C. M. Kewish, R. Wepf, O. Bunk, and F. Pfeiffer, Nature (London) 467, 436 (2010).

[4] Y. Takahashi, A. Suzuki, N. Zettsu, Y. Kohmura, Y. Senba, H. Ohashi, K. Yamauchi, and T. Ishikawa, Phys. Rev. B 83, 214109 (2011).

[5] J. Vila-Comamala, A. Diaz, M. Guizar-Sicairos, A. Mantion, C. M. Kewish, A. Menzel, O. Bunk, and C. David, Opt. Express 19, 21333 (2011).

[6] A. Schropp, R. Hoppe, J. Patommel, D. Samberg, F. Seiboth, S. Stephan, G. Wellenreuther, G. Falkenberg, and C. G. Schroer, Appl. Phys. Lett. 100, 253112 (2012).

[7] M. Holler, A. Diaz, M. Guizar-Sicairos, P. Karvinen, E. Färm, E. Härkönen, M. Ritala, A. Menzel, J. Raabe, and O. Bunk, Sci. Rep. 4, 3857 (2014).

[8] A. Schropp, R. Hoppe, V. Meier, J. Patommel, F. Seiboth, H. J. Lee, B. Nagler, E. C. Galtier, B. Arnold, U. Zastrau, J. B. Hastings, D. Nilsson, F. Uhlén, U. Vogt, H. M. Hertz, and C. G. Schroer, Sci. Rep. 3, 1633 (2013).

[9] D. J. Batey, S. Cipiccia, F. Van Assche, S. Vanheule, J. Vanmechelen, M. N. Boone, and C. Rau, Sci. Rep. 9, 12278 (2019).

[10] S. Marchesini, H. Krishnan, B. J. Daurer, D. A. Shapiro, T. Perciano, J. A. Sethian, and F. R. N. C. Maia, J. Appl. Crystallogr. 49, 1245 (2016).

[11] B. Enders and P. Thibault, Proc. R. Soc. A 472, 20160640 (2016).
[12] A. M. Maiden and J. M. Rodenburg, Ultramicroscopy 109, 1256 (2009).

[13] M. Guizar-Sicairos and J. R. Fienup, Opt. Express 16, 7264 (2008).

[14] K. Wakonig, H.-C. Stadler, M. Odstrcil, E. H. R. Tsai, A. Diaz, M. Holler, I. Usov, J. Raabe, A. Menzel, and M. Guizar-Sicairos, J. Appl. Crystallogr. 53, 574 (2020).

[15] M. Wansleben, C. Zech, C. Streeck, J. Weser, C. Genzel, B. Beckhoff, and R. Mainz, J. Anal. At. Spectrom. 34, 1497 (2019).

[16] M. J. Derry, O. O. Mykhaylyk, A. J. Ryan, and S. P. Armes, Chem. Sci. 9, 4071 (2018).

[17] H. M. Thirimanne, K. D. G. I. Jayawardena, A. J. Parnell, R. M. I. Bandara, A. Karalasingam, S. Pani, J. E. Huerdler, D. G. Lidzey, S. F. Tedde, A. Nisbet, C. A. Mills, and S. R. P. Silva, Nat. Commun. 9, 2926 (2018).

[18] A. Bjeoumikhov, G. Buzanich, N. Langhoff, I. Ordavo, M. Radtke, U. Reinholz, H. Riesemeier, O. Scharf, H. Soltau, and R. Wedell, J. Instrum. 7, C11008 (2012).

[19] O. Scharf, S. Ihle, I. Ordavo, V. Arkadiev, A. Bjeoumikhov, S. Bjeoumikhova, G. Buzanich, R. Gubzhokov, A. Gunther, R. Hartmann, M. Kuhbacher, M. Lang, N. Langhoff, A. Liebel, M. Radtke, U. Reinholz, H. Riesemeier, H. Soltau, L. Struder, A. F. Thunemann, and R. Wedell, Anal. Chem. 83, 2532 (2011).

[20] Applications and X-ray techniques, https://web.archive.org/ 20201204165405/http://www.excillum.com/applications/ (2020), accessed: 2021-02-01.

[21] M. Otendal, T. Tuohimaa, U. Vogt, and H. M. Hertz, Rev. Sci. Instrum. 79, 016102 (2008).

[22] I. Ordavo, S. Ihle, V. Arkadiev, O. Scharf, H. Soltau, A. Bjeoumikhov, S. Bjeoumikhova, G. Buzanich, R. Gubzhokov, A. Günther, R. Hartmann, P. Holl, N. Kimmel, M. Kühbacher, M. Lang, N. Langhoff, A. Liebel, M. Radtke, U. Reinholz, H. Riesemeier, G. Schaller, F. Schopper, L. Strüder, C. Thamm, and R. Wedell, Nucl. Instrum. Methods Phys. Res., Sect. A 654, 250 (2011).

[23] F. Van Assche, S. Vanheule, L. Van Hoorebeke, and M. N. Boone, Sensors 21, 563 (2021).

[24] F. V. Assche, S. Vanheule, S. Cipiccia, L. Vincze, L. V. Hoorebeke, and M. Boone, J. Instrum. 13, C11015 (2018).

[25] S. H. Nowak, A. Bjeoumikhov, J. von Borany, J. Buchriegler, F. Munnik, M. Petric, M. Radtke, A. D. Renno, U. Reinholz, O. Scharf, and R. Wedell, J. Anal. At. Spectrom. 30, 1890 (2015).

[26] D. J. Batey, Ptychographic imaging of mixed states, Thesis, University of Sheffield, 2014.

[27] A. M. Maiden, M. J. Humphry, M. C. Sarahan, B. Kraus, and J. M. Rodenburg, Ultramicroscopy 120, 64 (2012).

[28] D. J. Batey, D. Claus, and J. Rodenburg, Ultramicroscopy 138, 13 (2014).

[29] P. Thibault and A. Menzel, Nature (London) 494, 68 (2013).

[30] M. van Heel and M. Schatz, J. Struct. Biol. 151, 250 (2005).

[31] J. C. H. Spence, U. Weierstall, and M. Howells, Ultramicroscopy 101, 149 (2004).

[32] M. Eckermann, M. Tpperwien, A.-L. Robisch, F. van der Meer, C. Stadelmann, and T. Salditt, Journal of medical imaging 7, 1 (2020). 
[33] M. Dierick, D. Van Loo, B. Masschaele, J. Van den Bulcke, J. Van Acker, V. Cnudde, and L. Van Hoorebeke, Nucl. Instrum. Methods Phys. Res., Sect. B 324, 35 (2014).

[34] J. Van den Bulcke, M. Boone, J. Van Acker, M. Stevens, and LucVan Hoorebeke, Ann. Forest Sci. 66, 508 (2009).

[35] M. Endrizzi, F. A. Vittoria, P. C. Diemoz, R. Lorenzo, R. D. Speller, U. H. Wagner, C. Rau, I. K. Robinson, and A. Olivo, Opt. Lett. 39, 3332 (2014).

[36] B. Gunther, R. Gradl, C. Jud, E. Eggl, J. Huang, S. Kulpe, K. Achterhold, B. Gleich, M. Dierolf, and F. Pfeiffer, J. Synchrotron Radiat. 27, 1395 (2020).

[37] S. Cipiccia, M. R. Islam, B. Ersfeld, R. P. Shanks, E. Brunetti, G. Vieux, X. Yang, R. C. Issac, S. M. Wiggins, G. H. Welsh, M.-P. Anania, D. Maneuski, R. Montgomery, G. Smith, M. Hoek, D. J. Hamilton, N. R. C. Lemos, D.
Symes, P. P. Rajeev, V. O. Shea, J. M. Dias, and D. A. Jaroszynski, Nat. Phys. 7, 867 (2011).

[38] G. P. Williams, Nucl. Instrum. Methods Phys. Res., Sect. A 266, 59 (1988).

[39] X. F. D. Stragier, P. H. A. Mutsaers, and O. J. Luiten, Microsc. Microanal. 24, https://doi.org/10.1017/S14319276 18013880.

[40] M. Hirose, T. Higashino, N. Ishiguro, and Y. Takahashi, Opt. Express 28, 1216 (2020).

[41] S. C. Mayo, P. R. Miller, S. W. Wilkins, T. J. Davis, D. Gao, T. E. Gureyev, D. Paganin, D. J. Parry, A. Pogany, and A. W. Stevenson, J. Microsc. 207, 79 (2002).

[42] P. Stahlhut, T. Ebensperger, S. Zabler, and R. Hanke, J. Phys. Conf. Ser. 463, 012007 (2013).

[43] A. Tkachuk, F. Duewer, H. Cui, M. Feser, S. Wang, and W. Yun, Z. Kristallogr. 222, 650 (2007). 